\title{
Multiorgan accelerated silicosis misdiagnosed as sarcoidosis in two workers exposed to quartz conglomerate dust
}

\author{
Gabriella Guarnieri, ${ }^{1}$ Rosana Bizzotto, ${ }^{2}$ Ottorino Gottardo, ${ }^{2}$ Emanuela Velo, ${ }^{3}$ \\ Mauro Cassaro, ${ }_{1}^{4}$ Stefania Vio, ${ }^{5}$ Maria Grazia Putzu, ${ }^{6}$ Federica Rossi, ${ }^{6}$ Paolo Zuliani, \\ Filippo Liviero, ${ }^{1}$ Paola Mason, ${ }^{1}$ Piero Maestrelli ${ }^{1}$
}

${ }^{1}$ Department of Cardiologic, Thoracic and Vascular Sciences, University of Padova, Padova, Italy

${ }^{2}$ SPISAL - ULSS6 Euganea,

Padova, Italy

${ }^{3}$ Medicine Unit, ULSS 6 Euganea, Camposampiero, Italy ${ }^{4}$ Pathology Unit, ULSS 6 Euganea, Camposampiero, Italy ${ }^{5}$ Radiology Unit, University of Padova, Padova, Italy

${ }^{6}$ Department of Public Health and Pediatric Sciences, University of Turin, Turin, Italy

Correspondence to Professor Piero Maestrelli, Department of Cardiologic, Thoracic and Vascular Sciences, University of Padova, Padova 35128, Italy;

piero.maestrelli@unipd.it

Received 29 August 2018 Revised 7 November 2018 Accepted 12 November 2018 Published Online First 4 December 2018

\section{ABSTRACT}

Introduction Clusters of silicosis cases have been reported in the fabrication of quartz conglomerate, a new high-silica-content artificial stone for kitchen and bathroom benchtops (countertops).

Aim We describe two cases of accelerated-type silicosis with hepatic granulomas arising in workers exposed to artificial quartz conglomerates.

Methods A confident diagnosis of multiorgan silicosis was based on high level of respirable silica in the workplace, typical radiological alterations in chest high-resolution $C T$, histological findings in the lung and liver, and detection of silica crystals in both tissues by phase-contrast polarising light microscopy and scanning electron microscopy and energy dispersive spectroscopy.

Results The development of the disease $<10$ years after the first exposure is consistent with an acceleratedtype of silicosis. Compared with other studies related to quartz conglomerate exposure, we determined that the levels of airborne crystalline silica during activity in the finishing area were between 0.260 and $0.744 \mathrm{mg} /$ $\mathrm{m}^{3}$, that is, much higher than the threshold limit value according to American Conference of Governmental Industrial Hygienists $\left(0.025 \mathrm{mg} / \mathrm{m}^{3}\right)$. Moreover, liver granulomas were associated with accumulation of crystalline silica particles in the hepatic tissue.

Conclusions Quartz conglomerate fabrication is a potentially dangerous occupation. General practitioners and physicians should have awareness of this newly described occupational hazard. Accurate occupational history is critical in avoiding misdiagnosis, as silicosis caused by inhalation of dust from artificial quartz conglomerates may exhibit atypical presentation. These features seem to be related to the extremely high level of silica exposure and, possibly, to an increased toxicity of the dust generated in this process.

\section{INTRODUCTION}

(C) Author(s) (or their employer(s)) 2019. No commercial re-use. See rights and permissions. Published by BMJ.

To cite: Guarnieri G, Bizzotto R, Gottardo O, et al. Occup Environ Med 2019;76:178-180.
Clusters of silicosis cases related to the fabrication of quartz conglomerates, a new high-silica content (70\%-90\%) artificial stone for kitchen and bathroom benchtops (countertops), have been recently described. ${ }^{1-4}$ We reported two cases of multiorgan silicosis in workers exposed to quartz conglomerates, who received initially an incorrect diagnosis of sarcoidosis.

\section{Key messages}

What is already known about this subject?

- Clusters of silicosis cases have been reported in the fabrication of quartz conglomerate, a new high-silica content artificial stone for kitchen and bathroom benchtops.

What are the new findings?

- We report the first cases of accelerated silicosis in workers exposed to quartz conglomerates with liver involvement.

- Compared with other studies related to quartz conglomerate exposure, we provided data on the levels of workplace respirable silica exposure and demonstrated accumulation of crystalline silica particles in the lung and liver.

How might this impact on policy or clinical practice in the foreseeable future?

- General practitioners and physicians should have awareness of this newly described occupational risk.

- Accurate occupational history is critical in avoiding misdiagnosis, as silicosis caused by inhalation of dust from artificial quartz conglomerates may exhibit atypical presentation, that is, occurrence in young workers, predominant localisation in lung lymph nodes and extrapulmonary involvement.

- Actions are needed to reduce the risk of silicosis in this job.

\section{METHODS}

Between 2016 and 2017, two subjects with the previous diagnosis of sarcoidosis were referred to our Department of Occupational Medicine by their chest physicians after a poor response to treatment. The diagnosis of pulmonary and hepatic silicosis was obtained with: chest high-resolution CT (HRCT); pulmonary function tests; lung and liver biopsies; analysis of silica particles and metals in the tissues with phase contrast polarising light microscopy and scanning electron microscopy and energy dispersive spectroscopy. 


\section{RESULTS}

\section{Case 1}

A 35 -year-old man, former smoker (14 pack-year) presented with symptoms of sarcoidosis. He has worked in the finishing area of an ornamental stone company of Venetian region, with approximately 200 employees, since 2003. His duties consisted of cutting and polishing kitchen benchtops. Quartz conglomerate had been extensively used since 2008. He had been hospitalised on two occasions and treated with systemic prednisone for almost 2 years and then with methotrexate for 9 months, but he did not improve. A diagnosis of sarcoidosis was based on chest HRCT showing multiple bilateral enlarged lymph nodes without parenchymal alterations, negative serology for autoantibodies and QuantiFERON for Mycobacterium tuberculosis, increase of ACE (91U/L, normal range 8-52 U/L), abnormal hepatic function indices despite negative viral markers and $<1$ alcohol unit/day intake. Transmediastinal lung biopsies showed multiple inflammatory granulomas with a necrotic centre and giant cells, interstitial sclerohyalinosis, perivascular and peribronchial lymphocytic infiltrate and macrophages with cytoplasmatic pigments. Over approximately 3 years, the chest HRCT has changed and was consistent with silicosis (figure 1A). Pulmonary function test revealed a moderate restrictive pattern (total lung capacity, TLC 52\%) and reduction of carbon monoxide lung diffusion $\left(\mathrm{DL}_{\mathrm{CO}} 49 \%, \mathrm{~K}_{\mathrm{CO}} 80 \%\right)$. The liver biopsy showed portal polymorphic inflammatory infiltrate, numerous epithelioid microgranulomas and hepatocyte steatosis. We analysed the content of silica in lung and liver biopsies using optical and electron microscopies with evidence of numerous silica particles with a size of $0.5-4 \mu \mathrm{m}$.

\section{Case 2}

A 38-year-old man, former mild smoker (2 pack-year), presented with a 1-year history of abdominal symptoms and weight loss. He has worked since 2008 in the same company with the same
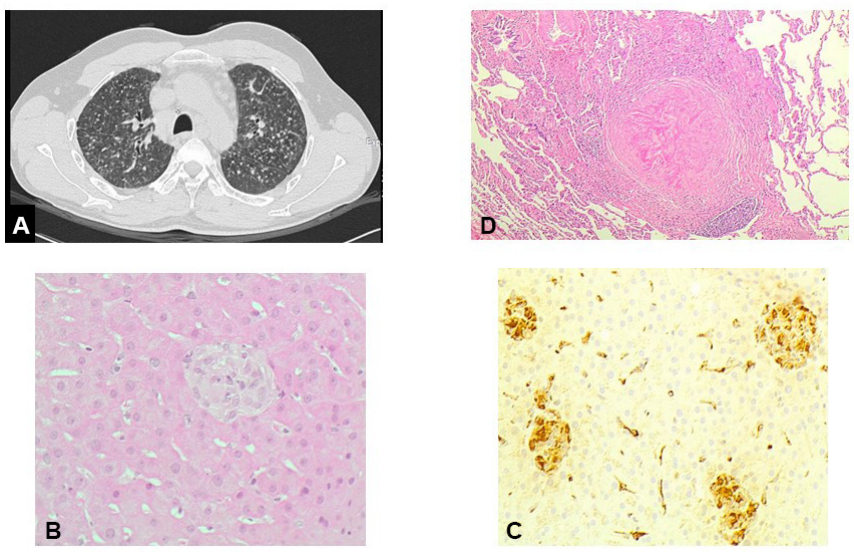

Figure 1 (A) Axial chest HRCT slice of patient 1: diffuse bilateral interstitial micronodular pattern; some patchy ground-glass opacities, more evident in the lower lung zone. ICOERD: prevalent well-defined rounded grade 2 opacities, predominantly size p, profusion 14 and irregular/linear opacities, grade 2 and predominantly intralobular. (B) Liver biopsy of case 2, H\&E stain (10x). (C) CD68 immunostaining of a liver biopsy of case 2 showing non-necrotising epithelioid granulomas of CD68-positive cells in a context of normal hepatic parenchyma. (D) Silicotic nodule in a lung biopsy of case 2 (H\&E stain, 10x), consisting of sclerohyaline centre and concentric laminated collagen fibres, surrounded by lymphocytes and giants cells. HRCT, high-resolution CT; ICOERD, International Classification of HRCT for Occupational and Environmental Respiratory Diseases. duties than the previous case. Hepatic function indices were abnormal, but viral markers were negative and he had $<1$ alcohol unit/day intake. Abdomen ultrasound revealed hepatosplenomegaly. The patient was admitted to a local hospital and after further investigations, a diagnosis of sarcoidosis was made. The liver biopsy revealed numerous non-necrotising epithelioid granulomas and centrilobular sinusoidal ectasia (figure 1B,C). Serology for autoantibodies was negative and ACE was increased (124U/L). Microbiology evaluation was negative. The chest HRCT was similar to that of case 1 shown in figure 1 and compatible with silicosis. Bronchoalveolar lavage fluid cell count showed $10 \%$ neutrophils, $85 \%$ macrophages and $5 \%$ of lymphocytes with a decreased CD4/CD8 ratio (0.5). Video-assisted lung surgical biopsies were obtained and the main histopathological findings were inflammatory granulomas with lymphocytic and giant cells infiltrate, and interstitial sclerohyalinosis without caseous necrosis (figure 1D). Pulmonary function test revealed a mild restrictive pattern (TLC 73\%) and mild reduction of carbon monoxide lung diffusion ( $\mathrm{DL}_{\mathrm{CO}} 63 \%$ predicted, $\mathrm{K}_{\mathrm{CO}} 77 \%$ ).

After 6 months of oral prednisone treatment without any improvement, the patient was re-evaluated in our centre. The analysis of lung and liver biopsies revealed numerous silica particles $(1-3 \mu \mathrm{m})$ in both tissues.

Both workers were supplied at work with Filtering Face Piece 2 (FFP2) masks and the finishing area had exhaust ventilation on the wall. However, the hygiene measurements proved ineffective. The levels of airborne crystalline silica, assessed by X-ray diffraction on samples taken according to NIOSH 7500:2003 during activity in the three finishing lines performed in 2016, showed 8 -hour average values of $0.260,0.486$ and $0.744 \mathrm{mg}$ $\mathrm{m}^{3}$, that is, 10 to 30 times higher than the threshold limit value according to American Conference of Governmental Industrial Hygienists (ACGIH) $\left(0.025 \mathrm{mg} / \mathrm{m}^{3}\right)$.

\section{DISCUSSION}

As far as we know, these are the first cases of lung and hepatic silicosis in workers exposed to artificial quartz conglomerates. The relatively young age, the radiological and histological aspect of the alterations, the initial overlooking of the work exposure had led first to an incorrect diagnosis of sarcoidosis. A confident diagnosis of silicosis was based on the high level of silica exposure, radiological and histological findings, and the detection of silica crystals in tissues. Compared with other studies related to quartz conglomerate exposure ${ }^{1-3}$ we provide data on levels of respirable silica in the workplace and demonstrate accumulation of silica particles in the lung and liver. The development of the disease $<10$ years after the first exposure is consistent with an accelerated-type of silicosis. ${ }^{5}$ Symptoms at presentation and elevated ACE levels might indicate a coexistence of sarcoidosis. However, it is unlikely that this relatively rare disease was present in both cases who developed silicosis on exposure to quartz conglomerate unless we admit that sarcoidosis can be caused by silica or other components in the dust. The former possibility was not corroborated by an epidemiological evidence ${ }^{6}$ and our data on metals in the tissue were inconsistent. In addition, drug treatment for sarcoidosis proved ineffective and the granulomas detected in the liver were not typical of sarcoidosis. ${ }^{7}$

Data from literature proved that exposure to dust from artificial quartz conglomerates caused several outbreaks of silicosis. Chronic silicosis was detected in approximately $3 / 4$ of these cases. Artificial conglomerates contain not only silica but also resins and pigments. Therefore, the possibility that different components may increase the toxicity of the dust in this process 
should be considered. In addition, freshly fractured silica, generated during abrasive blasting, may exhibit increased toxicity than aged silica, due to the elevated redox potential of silanol groups on crystal surface. ${ }^{8}$ No data on workplace dust levels were available from most of the previous studies on artificial quartz conglomerates, ${ }^{1-3}$ except the case report of Paolucci et $a l .{ }^{4}$ Their data in the finishing area showed an average crystalline silica concentration 20 times higher than the threshold limit value proposed by ACGIH. These levels of exposure to silica are similar to those measured in the workplace of our workers. In addition, the presentation as accelerated silicosis even in the two cases described by Paolucci et al ${ }^{4}$ suggests that a cumulative exposure to silica, sufficient to induce overt silicosis in $<10$ years, is reached in these working conditions.

Silicotic nodules are usually detected in hilar lymph nodes and lung parenchyma. The detection of silica in the liver does not necessarily implicate an extrapulmonary localisation of silicosis. However, it demonstrates that crystals able to induce silicosis were present in the tissue and this is in line with the finding of a hepatic involvement in autopsies of coal miners affected by anthracosilicosis described in the $80 \mathrm{~s} .{ }^{9-12}$ These authors defined a hepatic localisation of silicosis based on macrophage agglomerates in the presence of birefringent crystals. Fibrohyaline evolution was detected in a minority of cases. None of the above investigations actually demonstrated silica particles in the liver tissue, except Kadas et al. They showed deposits of anthracotic pigments in the liver, without any fibrotic reaction, in one autopsy of anthracosilicosis and, for the first time, characterised silica in the liver by X-ray microanalysis. ${ }^{10}$ We confirmed, in vivo, that inhaled silica may localise in the liver and demonstrated that the size of the particles is similar in the lung and the liver $(0.5-4 \mu \mathrm{m})$. The reaction of hepatic parenchyma to silica was characterised by granulomas of CD68-positive cells, but without fibrosis, at the variance of the findings in the lung.

The mechanisms by which silica reach the liver is unclear. The size of silica particles is too large for a translocation from the lung into circulation. Alveolar macrophages have a high phagocytic capacity and are able to uptake silica crystals. ${ }^{8}$ The clearance of inhaled particles from the lung occurs by cell migration to regional lymph nodes and through the lymphatic system to the circulation, and eventually to the liver. Moreover, silica particles can be ingested after lung mucociliary clearance and subsequently reach the liver by intestinal absorption. ${ }^{13}$ Whether liver localisation is the rule after silica inhalation or it occurs only with a massive lung deposition, like that of coal miners in the 1980 s and of our cases, needs to be determined.
In conclusion, benchtop fabrication from quartz conglomerate is a potentially dangerous occupation. General practitioners and physicians should have a heightened awareness of this newly described occupational hazard. Accurate occupational history is critical in avoiding misdiagnosis, as silicosis caused by inhalation of dust from artificial quartz conglomerates may exhibit atypical presentation, that is, occurrence in young workers, predominant localisation in lung lymph nodes and extrapulmonary involvement. These features seem to be related to the extremely high level of silica exposure and, possibly, to an increased toxicity of the dust generated in this process.

Contributors Conception, literature search, hypotheses delineation, acquisition of the data or the analysis and interpretation of such information; writing the article or substantial involvement in its revision prior to submission: GG and PM. Acquisition, analysis and interpretation of data: All authors.

Funding The authors have not declared a specific grant for this research from any funding agency in the public, commercial or not-for-profit sectors.

Competing interests None declared.

Patient consent Obtained.

Provenance and peer review Not commissioned; externally peer reviewed.

\section{REFERENCES}

1 Pérez-Alonso A, Córdoba-Doña JA, Millares-Lorenzo JL, et al. Outbreak of silicosis in Spanish quartz conglomerate workers. Int J Occup Environ Health 2014;20:26-32.

2 Kramer MR, Blanc PD, Fireman E, et al. Artificial stone silicosis [corrected]: disease resurgence among artificial stone workers. Chest 2012;142:419-24.

3 Hoy RF, Baird T, Hammerschlag G, et al. Artificial stone-associated silicosis: a rapidly emerging occupational lung disease. Occup Environ Med 2018;75:3-5.

4 Paolucci V, Romeo R, Sisinni AG, et al. Silicosis in workers exposed to artificial quartz conglomerates: does it differ from chronic simple silicosis? Arch Bronconeumol 2015;51:e57-60.

5 NIOSH. DHHS Publication N. 2002-129. Washington DC.

6 Calvert GM, Rice FL, Boiano JM, et al. Occupational silica exposure and risk of various diseases: an analysis using death certificates from 27 states of the United States. Occup Environ Med 2003;60:122-9.

7 Lewis J. Histopathology of granulomatous liver disease. Clin Liver Dis 2018;11:77-80.

8 Leung CC, Yu ITS, Chen W. Silicosis. The Lancet 2012;379:2008-18.

9 Dirschmid K, Kieser J. Der Morphologie del leber bei der Anthrakosilicose. Leber Magen Darm 1980;10:115-8.

10 Kádas I, Szende B, Csikós A, et al. Extrapulmonary localisation of carbon and silica particles in anthracosilicosis. Med Lav 1984;75:404-6.

11 Barbazza R, Calabro S, Tessarin C, et al. Granulomatous involvement of the liver in anthracosilicosis. Appl Pathol 1986:4:276-81.

12 LeFevre ME, Green FH, Joel DD, et al. Frequency of black pigment in livers and spleens of coal workers: correlation with pulmonary pathology and occupational information. Hum Pathol 1982;13:1121-6.

13 Lee W, Ahn YS, Lee S, et al. Occupational exposure to crystalline silica and gastric cancer: a systematic review and meta-analysis. Occup Environ Med 2016;73:794-801. 